\title{
A Network Pharmacology-based Analysis of the Molecular Mechanism of Tanreqing in the Treatment of the Coronavirus Disease 2019 (COVID-19)
}

Liuliu Yang ( $\nabla$ yll1031@126.com )

Respiratory and Critical Care Medicine,The First Affiliated Hospital of Guangzhou University of Chinese Medicine $\llbracket$ Guangzhou, Guangdong $₫ C h i n a \rrbracket$ https://orcid.org/0000-0001-9656-5062

Minyong Wen

First College of Clinical Medicine,Guangzhou University of Chinese Medicine $\mathbb{G}$ Guangzhou,Guangdong $₫$ China

Wenjiang Zheng

Respiratory and Critical Care Medicine,The First Affiliated Hospital of Guangzhou University of Chinese Medicine $₫$ Guangzhou, Guangdong $₫ C h i n a \bigotimes$

Xiaohong Liu

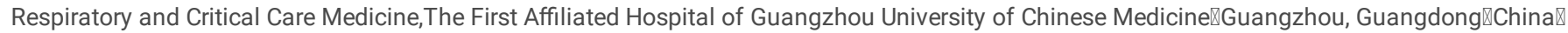
yong wang

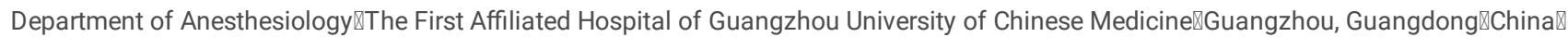

\section{Research}

Keywords: TRQ, Gene ontology, neuroactive ligand, Coronavirus disease 2019

Posted Date: August 10th, 2020

DOl: https://doi.org/10.21203/rs.3.rs-42041/v1

License: (c) (1) This work is licensed under a Creative Commons Attribution 4.0 International License. Read Full License 


\section{Abstract}

Background: This paper discusses the molecular mechanism of Tanreqing (TRQ) in the treatment of the coronavirus disease 2019 (COVID-19) using the network pharmacology approach. Our study provides new ideas on the laboratory research and clinical treatment of the disease.

Method: Information on the chemical constituents of TRQ and the genes targeted by the disease was collected. The common gene targets of the drug and the disease were input into the Search Tool for the Retrieval of Interacting Genes/Proteins $₫ S T R I N G \llbracket$ database to understand the interaction among target proteins. The protein-protein interaction (PPI) network and a network of the chemical constituents of TRQ and their targets were constructed using Cytoscape. Gene ontology (GO) enrichment analysis and Kyoto Encyclopedia of Genes and Genomes (KEGG) pathway analysis were performed using the R program and other relevant software packages.

Results: Twenty-eight active chemical constituents and 365 gene targets were identified for TRQ. Out of these genes, 113 were also found to be involved in the pathogenesis of the disease. Enrichment analysis revealed the therapeutic role that TRQ could have played in the treatment of COVID-19, via the regulation of important pathways such as the renin-angiotension system, neuroactive ligand-receptor interaction, phospholipase $D$ (PLD) signaling pathway, calcium signaling pathway, and the hypoxia-inducible factor 1 (HIF-1) signaling pathway.

Conclusions: This study attempts to predict the molecular mechanism of TRQ in the treatment of COVID-19, and suggests TRQ intervention through multiple targets and pathways in processes including inflammatory response, immune regulation, and apoptosis during the treatment of the disease. This study indicates the potential rational application of TRQ in the clinical treatment of COVID-19.

\section{Introduction}

Severe acute respiratory syndrome coronavirus 2 (SARS-CoV-2) is a novel coronavirus found in humans. The pneumonia caused by the infection of this virus is called "coronavirus disease 2019 (COVID-19)". SARS-CoV-2 is a betacoronavirus and the seventh coronavirus strain with known ability to infect humans. Its peers of the same species or genus, severe acute respiratory syndrome coronavirus (SARS-CoV,which caused the SARS outbreak in 2003)[1]and Middle East respiratory syndrome-related coronavirus (MERS-CoV,which caused the MERS outbreak in 2015)[2] have wreaked serious outbreaks of acute respiratory diseases in the world and caused panic among peoplein the past.[3]Ever since its first report in Wuhan, a city in the Hubei Province in central China, COVID-19 has spread to several countries in the world in just over two months. As of March 5, 2020, there were 95,917 confirmed cases globally.(The data came from National Health Commission of the People's Republic of China and World Health Organization). Currently, the exact pathogenesis of the disease is not yet fully understood by researchers, and an effective treatment using disease-specific drugs or prevention via vaccination is not available as yet. Traditional Chinese medicine (TCM) is a comprehensive medicine system with potential utilization value in clinical practice in China for thousands of years, and has the characteristics of multicomponents and multitargets, presenting synergistic effect on many diseases with fewer side effects.Its potential in the prevention and treatment of COVID-19 is gradually unfolding.

Based on the classification system of TCM, COVID-19 is an epidemic. Throughout the thousands of years of Chinese civilization, TCM has acquired rich clinical experience in the diagnosis and treatment of epidemics, and has been proven to reduce the mortality rate and improve the prognosis of diseases. In the Diagnosis and Treatment Program of COVID-19 (trial version 7), jointly issued by the National Health Commission and the National Administration of Traditional Chinese Medicine, People's Republic of China, Chinese herbal decoctions and Chinese patent medicine have been suggested as possible options for the treatment of COVID-19. Among them, Tanreqing (TRQ) is being recommended for severe and critical patients. TRQ is a type of compound Chinese medicine, commonly used in China. It consists of extracts from Radix Scutellariae baicalensis, bear bile powder, Cornu Caprae Hircus, Flos lonicerae, and Forsythiae Fructus. Because of its ability to clear heat, reduce phlegm, and eliminate toxic effects, this drug is often used to alleviate symptoms such as fever, cough, difficulty in expectoration, sore throat, dry mouth, reddish/crimson tongue, and yellow coating on tongue experienced in upper and lower respiratory tract infections and lung diseases[4-10]. TRQ, as a fast-acting and effective modern medication that comes in ready-to-use formulation, has been used increasingly in clinical situations. Though clinical studies and experiments in the past have confirmed TRQ as a natural broad-spectrum antiviral drug[5, 6, 11] the molecular mechanism behind its antiviral function has not yet been fully understood.

In terms of working mechanism, compound Chinese medicines regulate the human body comprehensively through multiple constituents-multiple targets interaction. It is, hence, difficult to clearly identify the mechanism of action of Chinese medicines. Network pharmacology is a classic method that uses bioinformatics to discover the potential mechanism of the interaction between drugs and known targets[12]. Its systematic and comprehensive approach agrees with the holistic characteristic of drug organization and matching in prescribing TCM. Network pharmacology has been widely used in the screening of active ingredients, identification of drug action mechanism, and the study of disease pathogenesis[13-17][18, 19]. This study sought to explore the possible material basis of TRQ action through network pharmacology, and lay the foundation for the investigation of the molecular basis of COVID-19 treatment by this drug.

\section{Materials And Methods}

\subsection{Chemical composition of TRQ}

Through literature research [11, 20-27]and using the list of chemical components supplied by the manufacturer (Shanghai Kaibao Pharmaceutical Co,Ltd,Shanghai,China),29 compounds in TRQ were chosen for further study. All compounds were fed into PubChem[28, 29]

(https://pubchem.ncbi.nlm.nih.gov). Compounds with duplicate data and no structural information were removed. Twenty-eight chemical constituents were eventually collected. 


\subsection{Prediction of the possible targets of TRQ}

The line notations (following the simplified molecular-input line-entry system, SMILES) of the 28 chemical constituents were imported into the Search Tool for Interacting Chemicals (STITCH) and the SwissTargetPrediction database. STITCH [30] (http://stitch.embl.de/) is a database of known and predicted interactions between chemical compounds and proteins. It uses text mining and molecular docking to predict the interactions between compounds and proteins. STITCH has been adopted in the research on TCM to locate potential active ingredients and in explaining its molecular mechanism [31].SwissTargetPrediction[32, 33](http://www.swisstargetprediction.ch/) is a web server that accurately predicts the similarity in the targets of bioactive molecules based on their chemistry. In the last step, the desired targets were obtained after removing the duplicate data.

\subsection{Targets involved in the pathogenesis of COVID-19 and those of TRQ in the treatment of COVID-19}

The targets involved in the pathogenesis of COVID-19 were obtained using single-cell sequencing. Angiotensin converting enzyme 2 (ACE2) has been identified as the receptor for the virus SARS-CoV-2. The interaction between ACE2 and the spike protein may also mediate the infection of the alveolar type /l cells in the lungs by SARS-CoV-2. The information on genescoexpressed with ACE2, as identified by the single-cell sequencing of colonic epithelial cells, was downloaded from that report [34]. The names of the genes obtained from the original file were standardized. Matching was performed using the Search Tool for the Retrieval of Interacting Genes/Proteins[35](STRING, https://string-db.org/) on human genes to obtain the potential targets associated with the disease (a total of 3558 of them). These gene targets were mapped to the targets of TRQ using the Venn diagram intersection to locate the overlapping targets of the drug and the virus, which are taken as the potential targets of TRQ action in the treatment of COVID-19 (drug-virus common targets).

\subsection{Network analysis}

The potential targets were fed into the STRING database, selecting "Homo sapiens" as the species. Results in the TSV format were exported and then imported into CytoHubba[36], a plug-in of Cytoscape [37]3.7.0for network analysis. The results obtained were the visualization of the network relationship among the top 20 targets (ranked by their degree values). A darker color on the chart indicates a higher degree value. Cytoscape is a software tool for bioinformatics analysis and is used for visualizing biological pathways and molecular interaction networks. CytoHubba is used for the ranking of the nodes in the network using its network analysis function. This plug-in is equipped with 11 topological analysis algorithms, which arebased on the degree of shortest path, edge percolated component, maximum neighborhood component, density of maximum neighborhood component, maximal clique centrality, and six centralities (bottleneck, eccentricity, closeness, radiality, betweenness, and stress).

\section{$2.5 \mathrm{GO}$ and pathway enrichment}

To understand the role of the potential targets in gene functions and signaling pathways, GO analysis [38] and Kyoto Encyclopedia of Genes and Genomes (KEGG) [39] pathway analysis were carried out using org.Hs.eg.db[40]and the clusterProfiler[41]package of R 3.5.2. clusterProfiler performs an automatic classification of biological terms and enrichment analysis of gene clusters. The R language (https://www.r-project.org/) and its back-end database "org.Hs.eg.db" were used to find the gene ID (entrezID) of the potential targets. Functional enrichment analysis using clusterProfiler was performed on the GO terms of these targets based on three aspects: biological process (BP), cellular component (CC), and molecular function (MF). The GO terms were then ranked according to their significance. The top 10 enriched terms were displayed on a histogram. In the same way, enrichment analysis was performed on KEGG pathways of the potential targets (gene ID [entrezID] retrieved) using the R language and the associated clusterProfiler package. The top 20 enriched terms were displayed on a histogram.

\section{Results}

\subsection{Screening of TRQ composition and targets}

Through literature search and using the list of chemical constituents supplied by the manufacturer, 29 compounds from the above five components of the drug were retrieved, including 4 from Radix Scutellariae baicalensis, 2 from bear bile powder, 13 from Cornu Caprae Hircus, 7 from Flos lonicerae, and 3 from Forsythiae Fructus. Because caffeic acid was present in both Flos loniceraeand Forsythiae Fructus, a total of 28 chemical constituents were obtained. The full list of chemical compounds present in TRQ is provided in Table 1. Also obtained were 365 potential targets for TRQ action. A chart depicting the interaction between the active constituents of the drug and the targets constructed using Cytoscape 3.7.0 is shown in Figure 1. The core compounds were obtained using NetworkAnalysis[35] for network topology Analysis. The top 10 core compounds (ranked by their degree values) are the following: ursodeoxycholic acid, caffeic acid, baicalin, 4,5-Di-O-caffeoylquinic acid, 3,4-Dicaffeoylquinic acid, chlorogenic acid , chenodeoxycholic acid, neochlorogenic acid, cryptochlorogenic acid, and amygdaloside.

Table 1 Chemical composition analysis of TRQ based on the constituent list provided by the manufacturer 


\begin{tabular}{|c|c|c|c|c|c|}
\hline Number & Medicinal material name & Constituent name & CAS number & Molecularformula & Molecular weight \\
\hline 1 & RadixScutellariaebaicalensis & Scutellarin & $27740-01-8$ & $\mathrm{C}_{21} \mathrm{H}_{18} \mathrm{O}_{12}$ & 462 \\
\hline 2 & Radix Scutellariaebaicalensis & Baicalin & 21967-41-9 & $\mathrm{C}_{21} \mathrm{H}_{18} \mathrm{O}_{11}$ & 446 \\
\hline 3 & Radix Scutellariaebaicalensis & Oroxylin A glucoronide & $36948-76-2$ & $\mathrm{C}_{5} \mathrm{H}_{8} \mathrm{O}_{6}$ & 164 \\
\hline 4 & Radix Scutellariaebaicalensis & Chrysin-7-0-glucuronide & $35775-49-6$ & $\mathrm{C}_{23} \mathrm{H}_{22} \mathrm{O}_{13}$ & 506 \\
\hline 5 & Floslonicerae & Neochlorogenic acid & $906-33-2$ & $\mathrm{C}_{16} \mathrm{H}_{18} \mathrm{O}_{9}$ & 354.31 \\
\hline 6 & Floslonicerae & Chlorogenic acid & $327-97-9$ & $\mathrm{C}_{16} \mathrm{H}_{18} \mathrm{O}_{9}$ & 354.31 \\
\hline 7 & Floslonicerae & Cryptochlorogenic acid & $905-99-7$ & $\mathrm{C}_{16} \mathrm{H}_{18} \mathrm{O}_{9}$ & 354.309 \\
\hline 8 & Floslonicerae & Caffeic acid & $331-39-5$ & $\mathrm{C}_{9} \mathrm{H}_{8} \mathrm{O}_{4}$ & 180.15 \\
\hline 9 & Floslonicerae & 3,4-Di-caffeoylquinic acid & $57378-72-0$ & $\mathrm{C}_{25} \mathrm{H}_{24} \mathrm{O}_{12}$ & 516.4509 \\
\hline 10 & Floslonicerae & 3,5-Di-caffeoylquinic acid & $2450-53-5$ & $\mathrm{C}_{25} \mathrm{H}_{24} \mathrm{O}_{12}$ & 516.4509 \\
\hline 11 & Floslonicerae & 4,5-Di-O-caffeoylquinic acid & $14534-61-3$ & $\mathrm{C}_{25} \mathrm{H}_{24} \mathrm{O}_{12}$ & 516.4509 \\
\hline 12 & Forsythiae Fructus & Forsythoside E & $93675-88-8$ & $\mathrm{C}_{20} \mathrm{H}_{30} \mathrm{O}_{12}$ & 462.4 \\
\hline 13 & Forsythiae Fructus & Caffeic acid & $331-39-5$ & $\mathrm{C}_{9} \mathrm{H}_{8} \mathrm{O}_{4}$ & 180.16 \\
\hline 14 & Forsythiae Fructus & Amygdaloside & $96420-61-0$ & $\mathrm{C}_{27} \mathrm{H}_{34} \mathrm{O}_{11}$ & 534.55 \\
\hline 15 & CornuCapraeHircus & Aspartic acid & $56-84-8$ & $\mathrm{C}_{4} \mathrm{H}_{7} \mathrm{NO}_{4}$ & 133.1 \\
\hline 16 & CornuCapraeHircus & L-Threonine & $72-19-5$ & $\mathrm{C}_{4} \mathrm{H}_{9} \mathrm{NO}_{3}$ & 119.12 \\
\hline 17 & CornuCapraeHircus & L-Alanine & $56-41-7$ & $\mathrm{C}_{3} \mathrm{H}_{7} \mathrm{NO}_{2}$ & 89.09 \\
\hline 18 & CornuCapraeHircus & Glutamic acid & $56-86-0$ & $\mathrm{C}_{5} \mathrm{H}_{9} \mathrm{NO}_{4}$ & 147.13076 \\
\hline 19 & CornuCapraeHircus & Arginine & $74-79-3$ & $\mathrm{C}_{6} \mathrm{H}_{14} \mathrm{~N}_{4} \mathrm{O}_{2}$ & 174.2 \\
\hline 20 & CornuCapraeHircus & DL-Methionine & $59-51-8$ & $\mathrm{C}_{5} \mathrm{H}_{11} \mathrm{O}_{2} \mathrm{NS}$ & 149.21 \\
\hline 21 & CornuCapraeHircus & Valine & 7004-03-7 & $\mathrm{C}_{5} \mathrm{H}_{11} \mathrm{NO}_{2}$ & 117.15 \\
\hline 22 & CornuCapraeHircus & Tyrosine & $60-18-4$ & $\mathrm{C}_{9} \mathrm{H}_{11} \mathrm{NO}_{3}$ & 181.189 \\
\hline 23 & CornuCapraeHircus & I-Isoleucine & $73-32-5$ & $\mathrm{C}_{6} \mathrm{H}_{13} \mathrm{NO}_{2}$ & 131.17 \\
\hline 24 & CornuCapraeHircus & Leucine & $61-90-5$ & $\mathrm{C}_{6} \mathrm{H}_{13} \mathrm{NO}_{2}$ & 131.18 \\
\hline 25 & CornuCapraeHircus & Phenylalanine & $63-91-2$ & $\mathrm{C}_{9} \mathrm{H}_{11} \mathrm{NO}_{2}$ & 165.19 \\
\hline 26 & CornuCapraeHircus & Histidine & $71-00-1$ & $\mathrm{C}_{6} \mathrm{H}_{9} \mathrm{~N}_{3} \mathrm{O}_{2}$ & 155 \\
\hline 27 & CornuCapraeHircus & L-Pyroglutamic acid & $98-79-3$ & $\mathrm{C}_{5} \mathrm{H}_{7} \mathrm{NO}_{3}$ & 129.12 \\
\hline 28 & bear bile powder & Ursodeoxycholic acid & $128-13-2$ & $\mathrm{C}_{24} \mathrm{H}_{40} \mathrm{O}_{4}$ & 392.572 \\
\hline 29 & bear bile powder & Chenodeoxycholic acid & $474-25-9$ & $\mathrm{C}_{24} \mathrm{H}_{40} \mathrm{O}_{4}$ & 395.57 \\
\hline
\end{tabular}

\subsection{Identification of potential drug targets}

Previous studies on COVID-19 have revealed ACE2 to be an essential protein involved in the viral infection of cells [42] $\mathbb{A}$ total of 3558 human genes that correlated with the expression of this protein were identified. The mapping of the TRQ targets with the SARS-CoV-2 targets yielded 113 genes as the possible core targets of the TRQ action in the treatment of the disease (Figure 2). It is thus believed that TRQ may affect the 113 proteins encoded by these genes through the chemical compounds identified in Section 3.1, and, thus, play a role in the development and outcome of the disease.

\subsection{Protein-protein interaction (PPI) network of the potential targets}

In TCM, the treatment of a disease is through the synergistic effect of multiple constituents on multiple targets. To understand the mechanism of this synergy and the possible mechanism of TRQ in the treatment of COVID-19, it is necessary to understand the effect of its constituents on the target proteins associated 
with COVID-19 pathogenesis. The 113 potential core targets identified by overlapping the TRQ targets with those involved in the disease were fed into the STRING database. The output obtained in the TSV format was imported into the cycloHubba plug-in of Cytoscape 3.7.0 for network analysis. The results are shown in Table 2. The network relationship among the top 20 targets (ranked by their degree values) is shown in Figure 3 . The top 20 target proteins are the following: epidermal growth factor receptor (EGFR), caspase3(CASP3), epidermal growth factor(EGF), signal transducer and activator of transcription 3(STAT3), angiotensin converting enzyme(ACE), estrogen receptor 1 (ESR1), glucagon (GCG), myeloperoxidase (MPO), Factor II (F2), interleukin 17A (IL17A), Pglycoprotein (ABCB1), cytochrome P450 2C9 囚CYP2C9囚, forkhead box protein p3 (FOXP3), Ras homolog gene family,member A (RHOA), UDP-

glucuronosyltransferase 1-8 (UGT1A8), Type-1 angiotensin II receptor (AGTR1),Metabotropic glutamate receptor 5(GRM5), cytochrome P450 2C19 (CYP2C19), caspase 8 (CASP8), and maltase-glucoamylase (MGAM). These proteins are thus considered the core targets in the PPI network and are thus closely associated with the treatment mechanism of TRQ for COVID-19. It is likely that TRQ achieves its medicinal effect and treats COVID-19 by acting on these core targets. The human angiotensin-converting enzyme 2 (ACE 2) is found ubiquitously in all the organs of the human body in the endothelial cells of the arteries and veins and the smooth muscle cells of the arteries. It exists in abundance in the epithelial cells of human lungs and small intestine, and is considered to be the receptor of SARS-CoV[43-45] [Previous studies on SARS-CoV-2 also reveal this enzyme as an essential protein in the viral infection of cells [42]. The target proteins identified are also involved in inflammatory response, immunosuppression, and cell growth, proliferation, differentiation, apoptosis, and reconstruction.

Table 2 Core targets in the PPI network of COVID-19 treatment by TRQ

\begin{tabular}{|c|c|c|c|c|c|c|c|c|c|c|c|c|c|}
\hline NO & Target & MCC & DMNC & MNC & Degree & EPC & BottleNeck & EcCentricity & Closeness & Radiality & Betweenness & Stress & Clusteri \\
\hline 1 & EGFR & 16706 & 0.30 & 34 & 36 & 40.63 & 10 & 0.33 & 68.83 & 4.24 & 1454.57 & 7000 & 0.19 \\
\hline 2 & CASP3 & 18658 & 0.36 & 35 & 35 & 41.28 & 9 & 0.25 & 69.08 & 4.27 & 1220.93 & 6566 & 0.25 \\
\hline 3 & EGF & 5612 & 0.40 & 27 & 29 & 40.38 & 9 & 0.33 & 65.33 & 4.18 & 798.31 & 4402 & 0.27 \\
\hline 4 & STAT3 & 17088 & 0.42 & 28 & 28 & 39.90 & 8 & 0.25 & 64.25 & 4.13 & 513.59 & 3234 & 0.32 \\
\hline 5 & ACE & 1280 & 0.34 & 22 & 24 & 37.23 & 8 & 0.33 & 63.33 & 4.16 & 961.95 & 5054 & 0.24 \\
\hline 6 & ESR1 & 5007 & 0.34 & 23 & 24 & 36.99 & 10 & 0.25 & 61.58 & 4.06 & 662.82 & 3780 & 0.26 \\
\hline 7 & GCG & 611 & 0.27 & 23 & 24 & 34.67 & 5 & 0.33 & 62.17 & 4.09 & 973.26 & 4976 & 0.20 \\
\hline 8 & MPO & 1494 & 0.33 & 20 & 22 & 35.91 & 9 & 0.25 & 60.25 & 4.02 & 761.50 & 3794 & 0.23 \\
\hline 9 & $\mathrm{~F} 2$ & 1938 & 0.37 & 22 & 22 & 37.18 & 4 & 0.33 & 62.67 & 4.16 & 573.25 & 3474 & 0.31 \\
\hline 10 & IL17A & 398 & 0.32 & 17 & 17 & 34.11 & 1 & 0.25 & 55.67 & 3.85 & 165.86 & 1104 & 0.29 \\
\hline 11 & ABCB1 & 428 & 0.38 & 14 & 16 & 32.27 & 10 & 0.25 & 57.25 & 3.96 & 400.95 & 2356 & 0.29 \\
\hline 12 & CYP2C9 & 144 & 0.30 & 16 & 16 & 27.74 & 2 & 0.25 & 54.25 & 3.79 & 338.34 & 2204 & 0.28 \\
\hline 13 & FOXP3 & 436 & 0.40 & 15 & 15 & 32.27 & 1 & 0.25 & 54.08 & 3.79 & 123.69 & 734 & 0.38 \\
\hline 14 & RHOA & 3257 & 0.50 & 14 & 15 & 33.69 & 2 & 0.25 & 55.75 & 3.90 & 202.32 & 1236 & 0.42 \\
\hline 15 & UGT1A8 & 124 & 0.30 & 15 & 15 & 26.18 & 3 & 0.25 & 54.42 & 3.81 & 542.86 & 2702 & 0.29 \\
\hline 16 & AGTR1 & 400 & 0.38 & 14 & 14 & 30.70 & 1 & 0.33 & 56.17 & 3.94 & 200.85 & 1410 & 0.37 \\
\hline 17 & GRM5 & 144 & 0.52 & 6 & 14 & 24.77 & 2 & 0.25 & 52.75 & 3.74 & 396.59 & 1800 & 0.21 \\
\hline 18 & CYP2C19 & 125 & 0.32 & 13 & 14 & 25.38 & 3 & 0.25 & 52.83 & 3.75 & 367.36 & 1964 & 0.27 \\
\hline 19 & CASP8 & 13712 & 0.56 & 14 & 14 & 32.67 & 1 & 0.25 & 53.83 & 3.80 & 88.50 & 598 & 0.55 \\
\hline 20 & MGAM & 204 & 0.39 & 11 & 13 & 27.04 & 4 & 0.25 & 54.33 & 3.85 & 502.93 & 2054 & 0.29 \\
\hline
\end{tabular}

\subsection{GO and KEGG enrichment analysis}

To systematically elucidate the numerous mechanisms of TRQ action on COVID-19, GO and KEGG pathway enrichment analyses were performed using org.Hs.eg.db and the clusterProfiler package of R 3.5.2. The top 10 enriched GO terms in the BP, MF, and CC categories were selected (Figure 4). In the figure, the color transition from blue to red indicates the increasing $\mathrm{P}$ value and the increasing significance of a term. According to the results, the $\mathrm{GO}$ biological process (GO.BP) category mainly includes terms such as organic anion transport, regulation of blood vessel size and diameter, eicosanoid metabolic process, hormone metabolic process, steroid metabolic process, chemical signal-mediated regulation of systemic arterial blood pressure, and the regulation of vascular process in the circulatory system. The GO cellular component (GO.CC) category mainly includes terms such as vesicle lumen, side of membrane, cytoplasmic vesicle lumen, secretary granule lumen, external side of plasma membrane, apical part of cell, membrane raft, membrane region, and membrane microdomain. The GO molecular function (GO.MF) category mainly includes terms such as exopeptidase activity, carbonate dehydratase activity, carboxylic acid binding, organic acid binding, nuclear receptor activity, direct ligand-regulated sequence-specific DNA binding, and metallopeptidase activity. 
To find the BPs related to the target proteins, enrichment analysis was performed on KEGG pathways,and we selected the pathways with the greatest relevance for further study (Figure 5). From the results of our research, the enriched signaling pathways were found to be the rennin-angiotensin system (RAS), neuroactive ligand-receptor interaction, phospholipase D (PLD) signaling pathway, bile secretion, chemical carcinogenesis, arachidonic acid metabolism, HIF-1 signaling pathway, calcium signaling pathway,advanced glycosylation end products (AGEs)-receptor of AGEs (AGE-RAGE) signaling pathway, and NF-kappa B signaling pathway. The most enriched pathways were the neuroactive ligand-receptor interaction, PLD signaling pathway, calcium signaling pathway, RAS, and the HIF-1 signaling pathway.

Currently, the RAS signaling pathway is thought to be deeply involved in the pathogenesis of COVID-19. Past research suggests the important role of this pathway in cardiovascular diseases, neurodegenerative diseases, and acute lung injury[46-50].

\section{Discussion}

TRQ is a type of compound Chinese medicine commonly used in China with clinically proven effectiveness [4-10]. It is often applied in the treatment of upper and lower respiratory tract infections. Currently, clinical observation indicates good prognosis in most patients infected with SARS-CoV-2,who have mild symptoms. However, some patients develop a number of fatal complications, including organ failure, septic shock, pulmonary edema, severe pneumonia, and acute respiratory distress syndrome (ARDS) [51, 52].The use of TRQ has gained the approval of most medical professionals in China in the treatment of severe COVID-19 patients. In the Diagnosis and Treatment Program of COVID-19 (trial versions 6 and 7), jointly issued by the National Health Commission and the National Administration of Traditional Chinese Medicine, People's Republic of China, TRQ has been listed as one of the alternative treatment modalities for severe and critical cases in Western medicine; TRQ has also been recommended for the treatment of severe (syndrome of flaring heat in qifen and yingfen in TCM) and critical cases (syndrome of internal blockade and external collapse in TCM) by TCM during the clinical treatment of confirmed cases. As of March 13,2020, two new clinical trials have been registered to evaluate the effectiveness and safety of TRQ therapy for COVID-19.

The pathogenesis of COVID-19 is highly complex, and at present has no effective treatment. TCM might play a great role in the prevention and treatment of complex diseases COVID-19 because of its wide functional range and the ability to act on multiple targets. In this study, network-based pharmacological analysis is performed on the multiple components-multiple targets action of the TRQ to offer a systematic understanding on the potential therapeutic mechanism of the drug in the treatment of COVID-19.

In this study, 28 active constituents, 113 potential targets, and 20 signaling pathways were identified by network pharmacology in the treatment of COVID-19 by TRQ. The results of network pharmacology reflect adequately the holistic and systematic approach adopted by TCM in the treatment of the disease. In the multiple-target network, ursodeoxycholic acid, caffeic acid, baicalin, 4,5-Di-0-caffeoylquinic acid, 3,4-Dicaffeoylquinic acid, chlorogenic acid , chenodeoxycholic acid, neochlorogenic acid, cryptochlorogenic acid, and amygdaloside are considered key chemical compounds. Since ancient times, Radix Scutellariae baicalensis (called Huangqin, HQ in Chinese), has been regarded as a golden herb in China with extensive application[53].Baicalin, its key component, is found to exhibit anti-inflammatory, anti-oxidative, anti-cancer, and anti-viral functions [54]. The anti-oxidative and anti-inflammatory properties may be attributed its ability of scavenging reactive oxygen species (ROS) and suppressing the NF-kB activity to inhibit the activities of a number of inflammatory cytokines and chemokines (including monocyte chemoattractant protein 1 [MCP-1], nitric oxide synthetase, cyclooxygenase, lipoxygenase, cell adhesion molecules, tumor necrosis factor, and interleukin) [55]. Flos lonicerae (called Jinyinhua, JYH in Chinese), is an extremely used traditional ediblemedicinal herb. Pharmacological studies have already proved JYH ideal clinical therapeutic effects on inflammation and infectious diseases and prominent effects on multiple targets in vitro and in vivo, such as pro-inflammatory protein inducible nitric oxide synthase, toll-like receptor 4, interleukin-1 receptor. [56]Caffeic acid, 4,5-Di-O-caffeoylquinic acid, 3,4-Dicaffeoylquinic acid, chlorogenic acid , neochlorogenic acid, and cryptochlorogenic acid are considered key chemical compounds of JYH.Caffeic acid and its derivatives have multiple biological activities, including antioxidants, antithrombosis, antihypertensive, antifibrosis, antiviral, and anti-tumor properties[57].Other active constituents of JYH, such as chlorogenic acid, also show antibacterial activity toward numerous organisms, including bacteria, yeast, mold, virus, and amoeba. The compounds also exhibit anti-oxidative, anti-inflammatory, lipid-lowering, antidiabetic, and anti-hypertensive functions [58]. Amygdaloside displays remarkable anti-inflammatory activity by suppressing the JAK-STAT and MAPK pathways and ROS production, inhibiting iNOS and COX-2 expression, and lowering TNF-a, IL-1 $\beta$, IL-6, NO, and PGE2[59, 60]. A variety of clinical studies have shown the beneficial effect of ursodeoxycholic acid in liver disease worldwide. Besides the well-known mechanisms of action of ursodeoxycholic acid,several studies have demonstrated that it serves as a potential therapeutic agent for a number non-hepatobiliary disorders including cardiovascular disease, immune disorders, organ transplantation and other[61].

The top 20 targets (ranked by their degree values) were selected by network analysis. They are, respectively, EGFR, CASP3, EGF, STAT3, ACE, ESR1, GCG, MPO, F2, IL17A, ABCB1, CYP2C9, FOXP3, RHOA, UGT1A8, AGTR1, GRM5, CYP2C19, CASP8, and MGAM. In network pharmacology, these target proteins are considered the key targets of TRQ action in the treatment of COVID-19. They participate in immune regulation, regulation and clearance of inflammation, and the promotion of cell proliferation, apoptosis, and reconstruction. ACE2 has been presently identified as the main functional receptor of SARS-nCoV-2[42]. The virus invades the lung cells through the binding of the S protein on its envelope to ACE2 [62]. This downregulates the level of ACE2 and results in the imbalance of ACE2 and ACE in the lungs. An increase in the level of angiotensin II (Ang II) elevates the permeability of pulmonary capillaries, leading to pulmonary edema and the infiltration of inflammatory cytokines. The cascade reaction triggered by cytokine storm soon causes pulmonary edema and dyspnea, and even death in some cases. For this reason, it is imperative to regulate ACE and inhibit the production and release of certain inflammatory and immune cytokines in the treatment of COVID-19. For example, EGFR is a surface protein that binds to the epidermal growth factor and is expressed in many cell types of the lungs, including smooth muscle cells, endothelial cells, fibroblasts, and epithelial cells [63]. Its immunoreactivity induces the production of mucin in the airway and airway remodeling [64] \and triggers airway inflammation by activating intracellular signaling pathways [65]. Signal transducer and activator of transcription-3 (STAT-3) plays an important role in the development and maturation of tissue functions in vertebrates, including inflammation control and immunity [66]. The specific inhibition activated by STAT-3 leads to a reduction in IL-17 production [67]. ESR1 impacts the regulation of immune

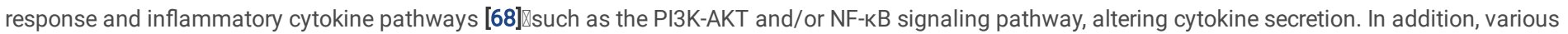


constituents of TCM are found to work on common targets, with the same molecule having an effect on different targets simultaneously. These findings indicate the synergy among multiple constituents of TRQ in regulating the virus targets.

Through GO enrichment analysis, we found that TRQ could regulate receptor activity, participate in hormone and steroid metabolism, and mediate BPs such the regulation of blood vessels, arterial blood pressure, and cytokine activity through chemical signals. The KEGG pathway enrichment analysis reveals the pathways that TRQ acts upon, including RAS, neuroactive ligand-receptor interaction, PLD signaling pathway, HIF-1 signaling pathway, calcium signaling pathway, AGE-RAGE signaling pathway, and NF-KB signaling pathway. RAS was found to mediate the production of pro-inflammatory cytokines, pro-fibrotic factors, and cellular free radicals, causing mitochondrial dysfunction and damage to tissue cells. It also was found to have a close relationship with the occurrence and development of inflammatory diseases. These effects and mechanisms of RAS have come to be known only recently and are gradually gaining attention. RAS, in particular renin, angiotensin II, and angiotensin-converting enzyme II (ACE2), play an important part in the onset, development, diagnosis, treatment, and prognosis of SARS [43, 69],influenza [47],and COVID-19 [42]. After binding to the receptor, angiotensin II activates signaling pathways such as the receptor-coupled phospholipase pathway, receptor-coupled tyrosine pathway, non-receptor-coupled tyrosine pathway, Rho pathway, and receptor-coupled active oxygen pathway. The renin-angiotensin Il-induced PLD pathway promotes cell hypertrophy, smooth muscle cell hyperplasia, and vasoconstriction. Through tyrosine phosphorylation, PLD activates MAPK and stimulates the production of growth factors and other cytokines, promoting cell mitosis and inflammation. The ATI receptor-coupled active oxygen pathway is closely related to vascular tension and cell hyperplasia, and is a key player in the production of inflammatory cytokines and the occurrence and development of ischemia-reperfusion injury, hypertension, and atherosclerosis. NF-KB is an important regulatory factor of inflammation and the immune system, and is involved in the transcription of the chemokine genes [70]. After its activation by IL17, the NF-KB signaling pathway promotes neutrophil recruitment, which could aggravate lung injury [71]. COVID-19 patients are also prone to reduced blood oxygen level due to lung inflammation. When this happens in the body, the hypoxia-induced regulatory network dominated by the hypoxia-inducible factor HIF1 will be activated. This network maintains the oxygen stability of all body cells and tissues and keeps them hypoxia-tolerant by adjusting the expression of related genes, regulating cell apoptosis. It is thus evident that TRQ may achieve a therapeutic effect on COVID-19 by regulating a number of signaling pathways and influencing pathological processes such as inflammatory response, apoptosis, and hypoxic injury.

\section{Conclusions}

Our research systematically investigated the possible mechanism of TRQ action, and found that it has the potential to act on COVID-19 through multiple constituents and pathways, involving interventions in inflammatory response, immune regulation, and apoptosis, so as to exert a therapeutic effect on the disease. The results of this study are consistent with the current findings on COVID-19. However, network pharmacology has limitations in its prediction. The active constituents, targets, and pathways proposed here need experimental evidence.

\section{Declarations}

\section{Data Availability}

Supporting data for the results of this study are available from the corresponding author.

\section{Conflicts of Interest}

The authors declare that they have no conflicts of interests.

\section{Authors' Contributions}

Liuliu Yang and Minyong Wen conceived and designed the research; Liuliu Yang,Wenjiang Zheng and Yong Wang collected and analyzed the data; Xiaohong Liu provided useful suggestions on the methodology; Liuliu Yang wrote the paper. All authors read and approved the final version of the manuscript.

\section{Acknowledgments}

This work was supported by the National Nature Science Foundation of China (Grant nos. 81904132 and 81973814).

\section{References}

1. C. Drosten, S. Gunther, W. Preiser, et al. "Identification of a novel coronavirus in patients with severe acute respiratory syndrome," N Engl J Med, vol. 348 , no. 20, pp. 1967-1976.

2. A.M. Zaki, S. van Boheemen, T.M. Bestebroer, A.D. Osterhaus, and R.A. Fouchier. "Isolation of a novel coronavirus from a man with pneumonia in Saudi Arabia," N Engl J Med, vol. 367, no. 19, pp. 1814-1820.

3. J. Cui, F. Li, and Z.L. Shi. "Origin and evolution of pathogenic coronaviruses," Nat Rev Microbiol, vol. 17, no. 3, pp. $181-192$.

4. H.L. Jiang, B. Mao, Y.Q. Zhong, H.M. Yang, and J.J. Fu. "[Tanreqing Injection for community-acquired pneumonia: a systematic review of randomized evidence]," Zhong Xi Yi Jie He Xue Bao, vol. 7, no. 1, pp. 9-19. 
5. P. Wang, X. Liao, Y.M. Xie, Y. Chai, and L.H. Li. "Tanreqing injection for acute bronchitis disease: A systematic review and meta-analysis of randomized controlled trials," Complement Ther Med, vol. 25, pp. 143-158.

6. P.Y. Xie, Y.M. Xie, L.X. Wang, Y.P. Chang, L. You, and X.L. Zhang. "[Registration study on analysis of adaptation syndromes and medication characteristics of tanreqing injection]," Zhongguo Zhong Yao Za Zhi, vol. 39, no. 18, pp. 3571-3575.

7. Y. Wang, T. Wang, J. Hu, et al. "Anti-biofilm activity of TanReQing, a Traditional Chinese Medicine used for the treatment of acute pneumonia," $J$ Ethnopharmacol, vol. 134, no. 1, pp. 165-170.

8. X.Y. Zhu, H.F. Xie, H. Han, and F. Gao. "[Network Meta-analysis of 3 kinds of Chinese medicine injections in treatment of acute exacerbations of chronic obstructive pulmonary disease]," Zhongguo Zhong Yao Za Zhi, vol. 44, no. 10, pp. 2179-2184.

9. X. Huang, X. Duan, Y. Zhu, K. Wang, J. Wu, and X. Tian. "Comparative efficacy of Chinese herbal injections for the treatment of community-acquired pneumonia: A Bayesian network meta-analysis of randomized controlled trials," Phytomedicine, vol. 63, p. 153009.

10. T. Pan, X. Liu, S. Xiang, and W. Ji. "Treatment for patients with multidrug resistant Acinetobacter baumannii pulmonary infection," Exp Ther Med, vol. 11, no. 4 , pp. 1345-1347.

11. H. Zhu, M. Chen, X. Shi, C. Shi, and C. Huang. "Material basis studies of anti-Influenza A active ingredients in Tanreqing Injection," Biomed Chromatogr, vol. 32 , no. 2.

12. Q. Luo, X. Shi, J. Ding, et al. "Network Pharmacology Integrated Molecular Docking Reveals the Antiosteosarcoma Mechanism of Biochanin A," Evid Based Complement Alternat Med, vol. 2019, p. 1410495.

13. S. Wei, X. Zhou, M. Niu, et al. "Network pharmacology exploration reveals the bioactive compounds and molecular mechanisms of Li-Ru-Kang against hyperplasia of mammary gland," Mol Genet Genomics, vol. 294, no. 5, pp. 1159-1171.

14. T. Qin, L. Wu, Q. Hua, Z. Song, Y. Pan, and T. Liu. "Prediction of the mechanisms of action of Shenkang in chronic kidney disease: A network pharmacology study and experimental validation," J Ethnopharmacol, vol. 246, p. 112128.

15. Y. Jiang, N. Liu, S. Zhu, X. Hu, D. Chang, and J. Liu. "Elucidation of the Mechanisms and Molecular Targets of Yiqi Shexue Formula for Treatment of Primary Immune Thrombocytopenia Based on Network Pharmacology," Front Pharmacol, vol. 10, p. 1136.

16. Y. Qu, Z. Zhang, Y. Lu, Zheng, and Y. Wei. "Network Pharmacology Reveals the Molecular Mechanism of Cuyuxunxi Prescription in Promoting Wound Healing in Patients with Anal Fistula," Evid Based Complement Alternat Med, vol. 2019, p. 3865121.

17. Z. Meng, X. Liu, J. Wu, et al. "Mechanisms of Compound Kushen Injection for the Treatment of Lung Cancer Based on Network Pharmacology," Evid Based Complement Alternat Med, vol. 2019, p. 4637839.

18. Y. Jiang, M. Zhong, F. Long, and R. Yang. "Deciphering the Active Ingredients and Molecular Mechanisms of Tripterygium hypoglaucum (Levl.) Hutch against Rheumatoid Arthritis Based on Network Pharmacology," Evid Based Complement Alternat Med, vol. 2020, p. 2361865.

19. H. Yang, Y. Li, S. Shen, et al. "Network Pharmacology-Based Investigation into the Mechanisms of Quyushengxin Formula for the Treatment of Ulcerative Colitis," Evid Based Complement Alternat Med, vol. 2019, p. 7870424.

20. C. Li, C. Zang, Q. Nie, B. Yang, B. Zhang, and S. Duan. "Simultaneous determination of seven flavonoids, two phenolic acids and two cholesterines in Tanreqing injection by UHPLC-MS/MS," J Pharm Biomed Anal, vol. 163, pp. 105-112.

21. S.X. Feng, X.H. Li, M.M. Wang, et al. "A sensitive HPLC-MS method for simultaneous determination of thirteen components in rat plasma and its application to pharmacokinetic study of Tanreqing injection," J Pharm Biomed Anal, vol. 148, pp. 205-213.

22. F. Zhang, L. Sun, S.H. Gao, W.S. Chen, and Y.F. Chai. "LC-MS/MS analysis and pharmacokinetic study on five bioactive constituents of Tanreqing injection in rats," Chin J Nat Med, vol. 14, no. 10, pp. 769-775.

23. S.Y. Liu, D.S. Xue, J.C. Pan, W.M. Zhang, W.L. Li, and H.B. Qu. "Screening and identification of multiple components in Tanreqing injection using RP-HPLC combined with DAD and ESI-TOF/MS," Chin J Nat Med, vol. 12, no. 7, pp. 535-541.

24. W. Li, X. Yan, J. Pan, S. Liu, D. Xue, and H. Qu. "Rapid analysis of the Tanreqing injection by near-infrared spectroscopy combined with least squares support vector machine and Gaussian process modeling techniques," Spectrochim Acta A Mol Biomol Spectrosc, vol. 218, pp. 271-280.

25. Y. Zhao, Z. Xu, T. Wang, et al. "Simultaneous quantitation of 23 bioactive compounds in Tanreqing capsule by high-performance liquid chromatography electrospray ionization tandem mass spectrometry," Biomed Chromatogr, vol. 33, no. 7, p. e4531.

26. W. Liu, X. Zhang, B. Mao, and H. Jiang. "Systems pharmacology-based study of Tanreqing injection in airway mucus hypersecretion," $J$ Ethnopharmacol, vol. 249 , p. 112425. 
27. W. Li, Z. Cheng, Y. Wang, and H. Qu. "A study on the use of near-infrared spectroscopy for the rapid quantification of major compounds in Tanreqing injection," Spectrochim Acta A Mol Biomol Spectrosc, vol. 101, pp. 1-7.

28. S. Kim. "Getting the most out of PubChem for virtual screening," Expert Opin Drug Discov, vol. 11, no. 9, pp. 843-855.

29. S. Kim, J. Chen, T. Cheng, et al. "PubChem 2019 update: improved access to chemical data," Nucleic Acids Res, vol. 47, no. D1, pp. D1102-D1109.

30. D. Szklarczyk, A. Santos, C. von Mering, L.J. Jensen, P. Bork, and M. Kuhn. "STITCH 5: augmenting protein-chemical interaction networks with tissue and affinity data," Nucleic Acids Res, vol. 44, no. D1, pp. D380-384.

31. M. Hong, S. Li, H.Y. Tan, et al. "A Network-Based Pharmacology Study of the Herb-Induced Liver Injury Potential of Traditional Hepatoprotective Chinese Herbal Medicines," Molecules, vol. 22, no. 4.

32. D. Gfeller, A. Grosdidier, M. Wirth, A. Daina, O. Michielin, and V. Zoete. "SwissTargetPrediction: a web server for target prediction of bioactive small molecules," Nucleic Acids Res, vol. 42, no. Web Server issue, pp. W32-38.

33. A. Daina, O. Michielin, and V. Zoete. "SwissTargetPrediction: updated data and new features for efficient prediction of protein targets of small molecules," Nucleic Acids Res, vol. 47, no. W1, pp. W357-W364.

34. J. Wang, S. Zhao, M. Liu, et al. "ACE2 expression by colonic epithelial cells is associated with viral infection, immunity and energy metabolism," medRxiv, p. 2020.2002.2005.20020545.

35. D. Szklarczyk, J.H. Morris, H. Cook, et al. "The STRING database in 2017: quality-controlled protein-protein association networks, made broadly accessible," Nucleic Acids Res, vol. 45, no. D1, pp. D362-D368.

36. C.H. Chin, S.H. Chen, H.H. Wu, C.W. Ho, M.T. Ko, and C.Y. Lin. "cytoHubba: identifying hub objects and sub-networks from complex interactome," BMC Syst Biol, vol. 8 Suppl 4, p. S11.

37. G. Su, J.H. Morris, B. Demchak, and G.D. Bader. "Biological network exploration with Cytoscape 3," Curr Protoc Bioinformatics, vol. 47 , pp. 813 11-24.

38. M. Ashburner, C.A. Ball, J.A. Blake, et al. "Gene ontology: tool for the unification of biology. The Gene Ontology Consortium," Nat Genet, vol. 25, no. 1, pp. 2529.

39. M. Kanehisa, S. Goto, S. Kawashima, Y. Okuno, and M. Hattori. "The KEGG resource for deciphering the genome," Nucleic Acids Res, vol. 32, no. Database issue, pp. D277-280.

40. C. Zhang, Y. Zheng, X. Li, X. Hu, F. Qi, and J. Luo. "Genome-wide mutation profiling and related risk signature for prognosis of papillary renal cell carcinoma," Ann Transl Med, vol. 7, no. 18, p. 427.

41. G. Yu, L.G. Wang, Y. Han, and Q.Y. He. "clusterProfiler: an R package for comparing biological themes among gene clusters," OMICS, vol. 16, no. 5, pp. 284287.

42. P. Zhou, X.L. Yang, X.G. Wang, et al. "A pneumonia outbreak associated with a new coronavirus of probable bat origin," Nature, vol. 579, no. 7798, pp. 270273.

43. X.Y. Ge, J.L. Li, X.L. Yang, et al. "Isolation and characterization of a bat SARS-like coronavirus that uses the ACE2 receptor," Nature, vol. 503, no. 7477, pp. 535-538.

44. W. Song, M. Gui, X. Wang, and Y. Xiang. "Cryo-EM structure of the SARS coronavirus spike glycoprotein in complex with its host cell receptor ACE2," PLoS Pathog, vol. 14, no. 8, p. e1007236.

45. K. Kuba, Y. Imai, S. Rao, et al. "A crucial role of angiotensin converting enzyme 2 (ACE2) in SARS coronavirus-induced lung injury," Nat Med, vol. 11, no. 8, pp. 875-879.

46. M. Vaillancourt, P. Chia, S. Sarji, et al. "Autonomic nervous system involvement in pulmonary arterial hypertension," Respir Res, vol. 18, no. 1, p. 201.

47. F. Huang, J. Guo, Z. Zou, et al. "Angiotensin II plasma levels are linked to disease severity and predict fatal outcomes in H7N9-infected patients," Nat Commun, vol. 5, p. 3595.

48. W.A. Hsueh and K. Wyne. "Renin-Angiotensin-aldosterone system in diabetes and hypertension," J Clin Hypertens (Greenwich), vol. 13, no. 4, pp. $224-237$.

49. M. Mogi and M. Horiuchi. "Effect of angiotensin II type 2 receptor on stroke, cognitive impairment and neurodegenerative diseases," Geriatr Gerontol Int, vol. 13 , no. 1 , pp. 13-18.

50. D. Shrikrishna, R. Astin, P.R. Kemp, and N.S. Hopkinson. "Renin-angiotensin system blockade: a novel therapeutic approach in chronic obstructive pulmonary disease," Clin Sci (Lond), vol. 123, no. 8, pp. 487-498. 
51. S.A. Rasmussen, J.C. Smulian, J.A. Lednicky, T.S. Wen, and D.J. Jamieson. "Coronavirus Disease 2019 (COVID-19) and Pregnancy: What obstetricians need to know," Am J Obstet Gynecol.

52. N. Chen, M. Zhou, X. Dong, et al. "Epidemiological and clinical characteristics of 99 cases of 2019 novel coronavirus pneumonia in Wuhan, China: a descriptive study," Lancet, vol. 395, no. 10223, pp. 507-513.

53. Q. Zhao, X.Y. Chen, and C. Martin. "Scutellaria baicalensis, the golden herb from the garden of Chinese medicinal plants," Sci Bull (Beijing), vol. 61, no. 18, pp. 1391-1398.

54. H.C. Zhou, R. Du, H. Wang, et al. "[Advance in studies on pharmacokinetics of baicalin]," Zhongguo Zhong Yao Za Zhi, vol. 43, no. 4, pp. 684-688.

55. B. Dinda, S. Dinda, S. DasSharma, R. Banik, A. Chakraborty, and M. Dinda. "Therapeutic potentials of baicalin and its aglycone, baicalein against inflammatory disorders," Eur J Med Chem, vol. 131, pp. 68-80.

56. Y. Li, W. Li, C. Fu, Y. Song, and Q. Fu. "Lonicerae japonicae flos and Lonicerae flos: a systematic review of ethnopharmacology, phytochemistry and pharmacology," Phytochem Rev, pp. 1-61.

57. M. Matejczyk, R. Swislocka, M. Kalinowska, G. Swidersk, W. Lewandowsk, and A. Jablonska-Trypuo. "Monitoring of Synergistic Enhancement of Caffeic Acid on Escherichia Coli K-12 Reca::Gfp Strain Treated with Dacarbazine," Acta Pol Pharm, vol. 74, no. 3, pp. $809-816$.

58. J. Santana-Galvez, L. Cisneros-Zevallos, and D.A. Jacobo-Velazquez. "Chlorogenic Acid: Recent Advances on Its Dual Role as a Food Additive and a Nutraceutical against Metabolic Syndrome," Molecules, vol. 22, no. 3.

59. J. Bao, R. Ding, L. Zou, et al. "Forsythiae Fructus Inhibits B16 Melanoma Growth Involving MAPKs/Nrf2/HO-1 Mediated Anti-Oxidation and AntiInflammation," Am J Chin Med, vol. 44, no. 5, pp. 1043-1061.

60. X. Pan, X. Cao, N. Li, et al. "Forsythin inhibits lipopolysaccharide-induced inflammation by suppressing JAK-STAT and p38 MAPK signalings and ROS production," Inflamm Res, vol. 63, no. 7, pp. 597-608.

61. V.G. Radchenko, P.V. Seliverstov, and S.I. Sitkin. "[New aspects of pharmacological effects of ursodeoxycholic acid]," Eksp Klin Gastroenterol, no. 8, pp. 4-10.

62. P. Zhou, X.-L. Yang, X.-G. Wang, et al. "Discovery of a novel coronavirus associated with the recent pneumonia outbreak in humans and its potential bat origin," bioRxiv, p. 2020.2001.2022.914952.

63. A. Habibovic, M. Hristova, D.E. Heppner, et al. "DUOX1 mediates persistent epithelial EGFR activation, mucous cell metaplasia, and airway remodeling during allergic asthma," JCI Insight, vol. 1, no. 18, p. e88811.

64. A.Z. El-Hashim, M.A. Khajah, W.M. Renno, et al. "Src-dependent EGFR transactivation regulates lung inflammation via downstream signaling involving ERK1/2, PI3Kdelta/Akt and NFkappaB induction in a murine asthma model," Sci Rep, vol. 7, no. 1, p. 9919.

65. K. Tsuchiya, T. Jo, N. Takeda, et al. "EGF receptor activation during allergic sensitization affects IL-6-induced T-cell influx to airways in a rat model of asthma," Eur J Immunol, vol. 40, no. 6, pp. 1590-1602.

66. E.J. Hillmer, H. Zhang, H.S. Li, and S.S. Watowich. "STAT3 signaling in immunity," Cytokine Growth Factor Rev, vol. 31, pp. 1-15.

67. J. Wienke, W. Janssen, R. Scholman, et al. "A novel human STAT3 mutation presents with autoimmunity involving Th17 hyperactivation," Oncotarget, vol. 6, no. 24, pp. 20037-20042.

68. X. Dong, M. Xu, Z. Ren, et al. "Regulation of CBL and ESR1 expression by microRNA-223p, 513a-5p and 625-5p may impact the pathogenesis of dust miteinduced pediatric asthma," Int J Mol Med, vol. 38, no. 2, pp. 446-456.

69. I. Hamming, W. Timens, M.L. Bulthuis, A.T. Lely, G. Navis, and H. van Goor. "Tissue distribution of ACE2 protein, the functional receptor for SARS coronavirus. A first step in understanding SARS pathogenesis," J Pathol, vol. 203, no. 2, pp. 631-637.

70. N.L. Wu, D.Y. Huang, H.N. Tsou, Y.C. Lin, and W.W. Lin. "Syk mediates IL-17-induced CCL20 expression by targeting Act1-dependent K63-linked ubiquitination of TRAF6," J Invest Dermatol, vol. 135, no. 2, pp. 490-498.

71. H.W. Song, C. Yang, W. Liu, X.W. Liu, Z. Liu, and F. Gao. "Interleukin-17A Plays the Same Role on Mice Acute Lung Injury Respectively Induced by Lipopolysaccharide and Paraquat," Inflammation, vol. 40, no. 5, pp. 1509-1519.

\section{Figures}




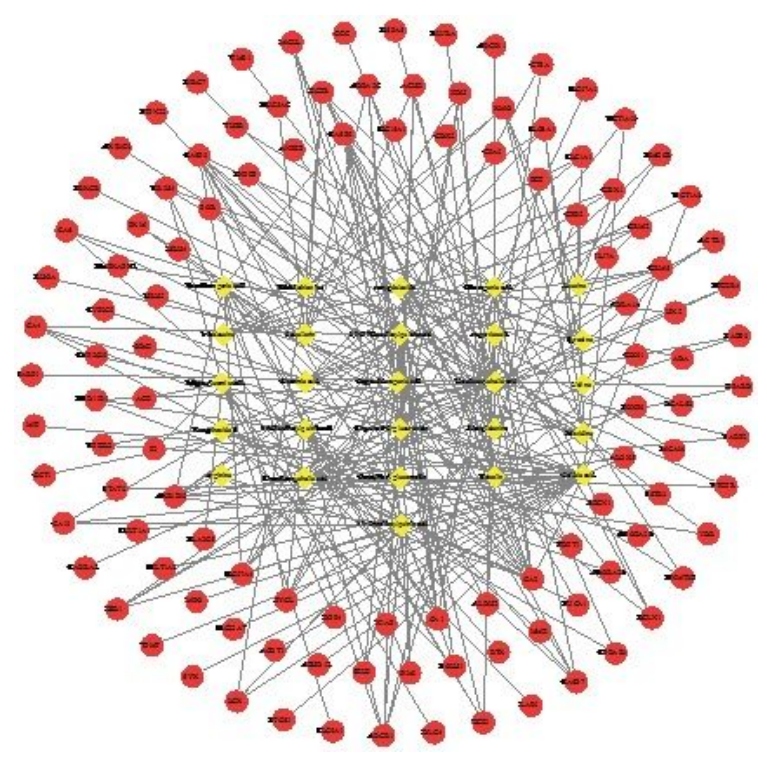

\section{Figure 1}

Interaction between active constituents and targets of TRQ (constructed using Cytoscape 3.7.0).

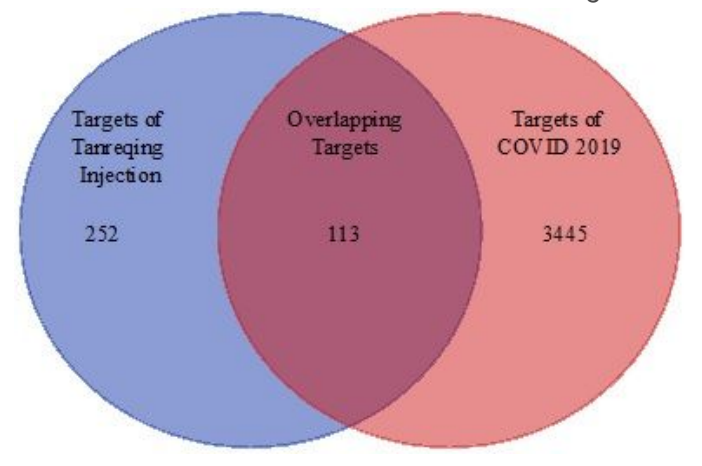

Figure 2

Venn diagram for the targets of TRQ active constituents and targets associated with COVID-19.

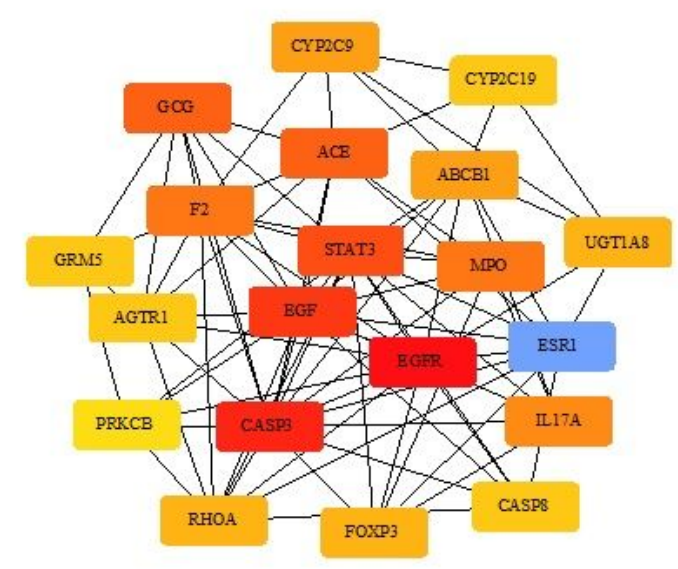

Figure 3

PPI network for the top 20 proteins as ranked by their degree values (retrieved in the STRING database and imported into Cytoscape for visualization; darker color indicates higher degree value. Detailed parameters are listed in Table 2). 


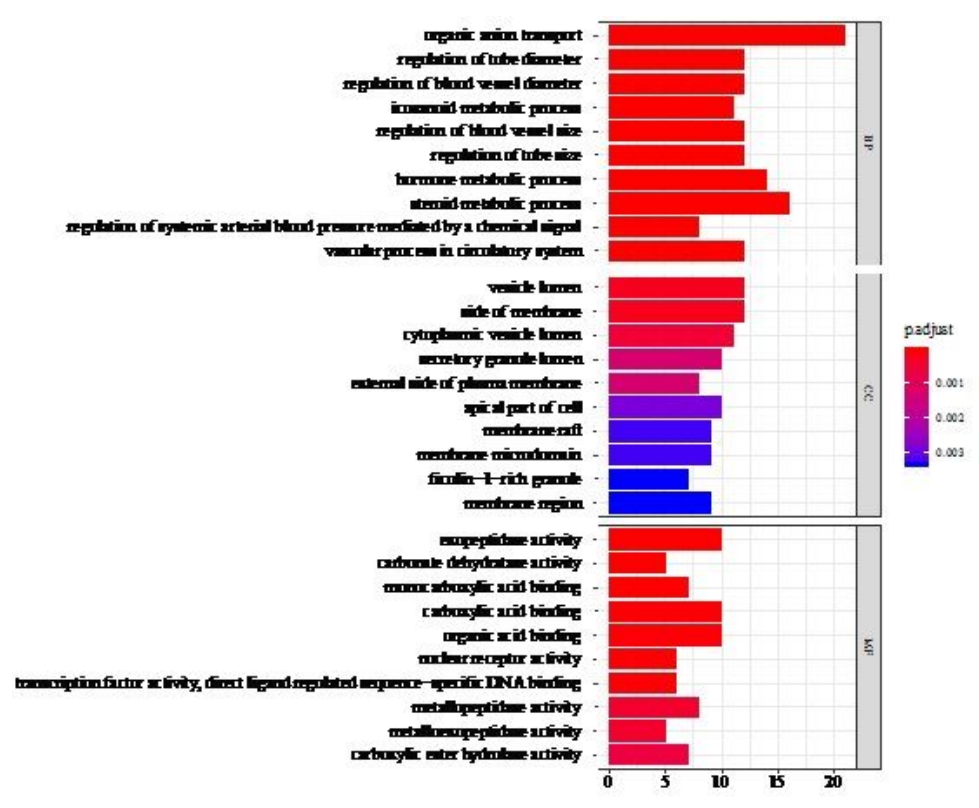

Figure 4

GO enrichment analysis ( $P<0.05$, performed with org. Hs.eg.db and clusterProfiler package of R 3.5.2).

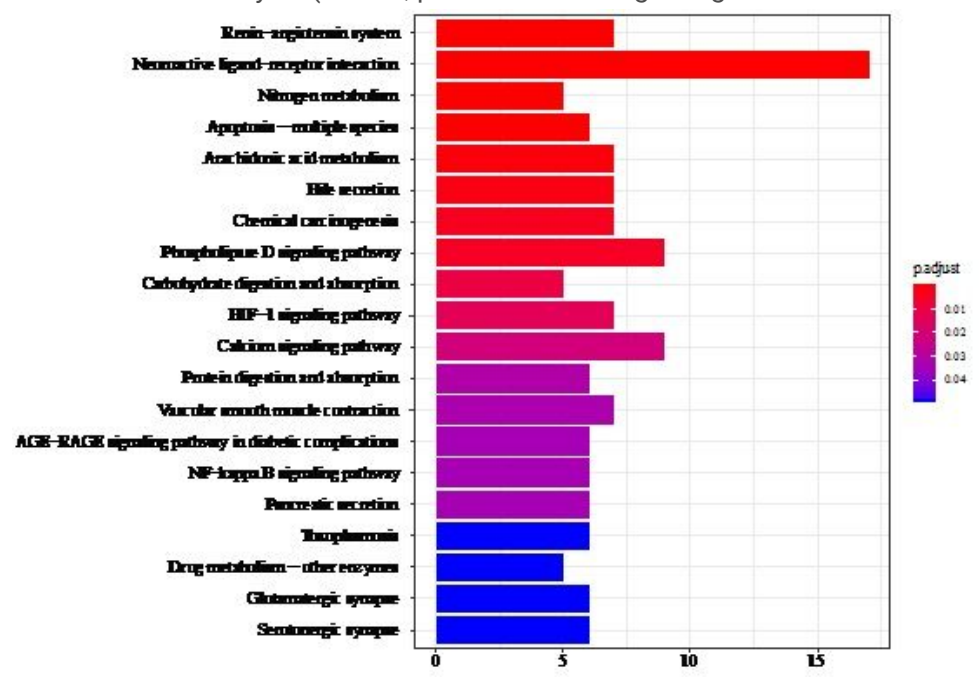

Figure 5

KEGG enrichment analysis ( $P<0.05$, performed with org.Hs.eg.db and clusterProfiler package of $R$ 3.5.2).

\section{Supplementary Files}

This is a list of supplementary files associated with this preprint. Click to download.

- SupplementaryMaterials.docx 